\title{
Measurable cardinals and fundamental groups of compact spaces
}

\author{
by \\ Adam Przeździecki (Warszawa)
}

\begin{abstract}
We prove that all groups can be realized as fundamental groups of compact spaces if and only if no measurable cardinals exist. If the cardinality of a group $G$ is nonmeasurable then the compact space $K$ such that $G=\pi_{1} K$ may be chosen so that it is path connected.

We construct a group whose cardinality equals the least measurable cardinal and which cannot be realized as the fundamental group of a compact Hausdorff space (Theorem 4.2). Since Keesling and Rudyak proved [6] that every group of smaller cardinality is the fundamental group of some compact space, we see that the negation of the large cardinal axiom about existence of measurable cardinals is equivalent to the statement that all groups can be obtained as fundamental groups of compact spaces. The last section gives an affirmative answer to the question, asked in [6], whether each group of nonmeasurable cardinality is the fundamental group of a path connected compact space.

All spaces considered below are completely regular, $I$ is the closed interval $[0,1]$ and $S^{1}$ is a circle. If $X$ is a space then we denote its Stone-Čech compactification by $\beta X$ and its Hewitt realcompactification by $v X$. If $f: X \rightarrow Y$ is a map then $\bar{f}$ denotes the induced map between the compactifications: $\beta X \rightarrow \beta Y$ or $v X \rightarrow v Y$.

A cardinal $\kappa$ is measurable if it admits a countably complete ultrafilter which is not fixed [4]. The least measurable cardinal is denoted by $\mathfrak{m}$ and the same symbol is used to denote the least ordinal and the discrete space of cardinality $\mathfrak{m}$. Note that $\mathfrak{m}$ is also the least measurable cardinal in the more restrictive sense [5, after 2.7] of admitting an $\mathfrak{m}$-complete ultrafilter.
\end{abstract}

2000 Mathematics Subject Classification: Primary 03E55; Secondary 55Qxx.

Key words and phrases: measurable cardinal, fundamental group.

The author was partially supported by grant 1 P03A 00526 . 
REMARK 0.1. The set $v \mathfrak{m} \backslash \mathfrak{m}$ is nonempty.

1. $\mathfrak{m}$-limits. In this section we introduce $\lambda$-limits ([3, §42], [7]) and prove Proposition 1.2 which states that if $G$ is the fundamental group of a compact space then $G$ admits m-limits. Additionally such limits commute with homomorphisms induced by maps between compact spaces.

LEMMA 1.1. If $Y$ is a locally compact, realcompact space of nonmeasurable cardinality, then $v(Y \times X)=Y \times v X$ for all $X$.

Proof. See [1, Corollary 2.2].

In this paper, Lemma 1.1 is applied for $Y$ compact.

Let $\lambda$ be an ordinal number. We say that a $\lambda$-limit is defined on a group $G$ if to each $\lambda$-sequence $\left(a_{\tau}\right)$ in $G$ there is assigned an element $a \in G$, denoted by

$$
a=\lim a_{\tau},
$$

subject to the following postulates:

(i) $\lim \left(a_{\tau} \cdot b_{\tau}\right)=\lim a_{\tau} \cdot \lim b_{\tau}$,

(ii) $\lim a_{\tau}=a$ if $a_{\tau}=a$ for all $\tau<\lambda$,

(iii) $\lim a_{\tau}=\lim b_{\tau}$ if $a_{\tau}=b_{\tau}$ for $\tau>\tau_{0}$ with some fixed $\tau_{0}<\lambda$.

Note that every $\lambda$-limit is a group homomorphism $\lim : G^{\lambda} \rightarrow G$. Our definition of a $\lambda$-limit is a direct generalization to nonabelian groups of the definition found in $[3, \S 42]$ and [7].

Proposition 1.2. For every point $s \in v \mathfrak{m} \backslash \mathfrak{m}$ there is an $\mathfrak{m}$-limit $\lim _{s}$ defined on fundamental groups of compact spaces.

Proof. Let $K$ be a compact space. Given an $\mathfrak{m}$-sequence $\left(a_{\tau}\right)$ in $\pi_{1} K$ we choose representatives $S^{1} \times\{\tau\}=S^{1} \rightarrow K$ of $a_{\tau}$ for $\tau<\mathfrak{m}$ and obtain a map $a: S^{1} \times \mathfrak{m} \rightarrow K$. It induces a map $v\left(S^{1} \times \mathfrak{m}\right) \rightarrow K$ which, by Lemma 1.1 , is a map $S^{1} \times v \mathfrak{m} \rightarrow K$. We restrict it to obtain a map

$$
\alpha: S^{1}=S^{1} \times\{s\} \rightarrow K
$$

which represents an element of $\pi_{1} K$. Two different representatives of the elements $a_{\tau}$ are connected by based homotopies, that is, maps $S^{1} \times I /\{*\} \times I$ $\rightarrow K$. Again by Lemma 1.1 and compactness of $S^{1} \times I /\{*\} \times I$, these maps produce a based homotopy between the respective $\alpha$ 's. Hence we have a well defined map $\lim _{s}$ which sends a sequence $\left(a_{\tau}\right)$ to an element of $\pi_{1} K$ represented by $\alpha$.

Verification of properties (i)-(iii) is straightforward.

Remark 1.3. The limits described in Proposition 1.2 commute with homomorphisms induced by continuous maps, that is, if $f: K \rightarrow L$ is a map 
between compact spaces then

$$
\lim _{s} f_{\#}\left(a_{\tau}\right)=f_{\#}\left(\lim _{s} a_{\tau}\right)
$$

for each $\mathfrak{m}$-sequence $\left(a_{\tau}\right)$ in $\pi_{1} K$.

2. Equal m-limits. In this section we prove (Proposition 2.2) that the fundamental group of a compact space which has a measurable cardinality admits nontrivial instances of equal $\mathfrak{m}$-limits.

LEMma 2.1. Let $D$ be a discrete space. For any point $s_{0} \in v D \backslash D$ there is a discrete space $X$ and functions $a, b: X \rightarrow D$ and an $s \in v X \backslash X$ such that $a(x) \neq b(x)$ for each $x \in X$ but $\bar{a}(s)=\bar{b}(s)=s_{0}$.

Proof. Let $\Delta=\{(d, d) \mid d \in D\}$ and $X=D \times D \backslash \Delta$. Since the closure of $\{d\} \times(D \backslash\{d\})$ in $v D \times v D$ is $\{d\} \times(v D \backslash\{d\})$ we see that the closure of $X$ is $v D \times v D \backslash \Delta$, in particular for each $s_{0} \in v D \backslash D$ the point $\left(s_{0}, s_{0}\right)$ is in the closure of $X$. We can define the functions $a$ and $b$ as the inclusion $X \rightarrow D \times D$ composed with the standard projections.

Proposition 2.2. If $K$ is a compact space and the cardinality of $G=$ $\pi_{1} K$ is measurable then there exist $\mathfrak{m}$-sequences $\left(a_{\tau}\right)$ and $\left(b_{\tau}\right)$ in $G$ and an $s \in v \mathfrak{m} \backslash \mathfrak{m}$ such that $a_{\tau} \neq b_{\tau}$ for all $\tau<\mathfrak{m}$ but $\lim _{s} a_{\tau}=\lim _{s} b_{\tau}$.

Additionally we may fix any two distinct elements $c$ and $d$ in $G$ and require that for each $\tau<\mathfrak{m}$ the sets $\left\{a_{\tau}, b_{\tau}\right\}$ and $\{c, d\}$ are disjoint.

Proof. Let $D \subseteq G$ be a subset of cardinality $\mathfrak{m}$, disjoint from $\{c, d\}$. We represent the elements of $D$ by a map $S^{1} \times D \rightarrow K$ where $D$ is treated as a discrete space. This leads to the following sequence of maps:

$$
\begin{array}{r}
S^{1} \times v \mathfrak{m} \rightarrow S^{1} \times v(D \times D) \rightarrow S^{1} \times v D \times v D \rightarrow S^{1} \times S^{1} \times v D \times v D \\
=v\left(S^{1} \times D\right) \times v\left(S^{1} \times D\right) \rightarrow K \times K .
\end{array}
$$

The first map is the identity on $S^{1}$ times the Hewitt realcompactification applied to the map in Lemma 2.1 with $X=\mathfrak{m}$. The second map is induced by a realcompactification of the inclusion $D \times D \rightarrow v D \times v D$, and the third one by the diagonal $S^{1} \rightarrow S^{1} \times S^{1}$. The equality is induced by the homeomorphism described in Lemma 1.1. The last map is the product of two copies of the realcompactification of the map $S^{1} \times D \rightarrow K$.

We choose an $s \in v \mathfrak{m} \backslash \mathfrak{m}$ as in Lemma 2.1. The composition (2.3) restricted to $S^{1} \times\{\tau\}$ represents a pair $\left(a_{\tau}, b_{\tau}\right)$ for some distinct $a_{\tau}$ and $b_{\tau}$ in $G$. Restriction of $(2.3)$ to $S^{1} \times\{s\}$ represents $\left(\lim _{s} a_{\tau}, \lim _{s} b_{\tau}\right)$. Since the image of $s$ in $v D \times v D$ is $\left(s_{0}, s_{0}\right)$ we see that $\lim _{s} a_{\tau}=\lim _{s} b_{\tau}$.

3. Examples. In this section we construct groups of measurable cardinality which cannot be realized as fundamental groups of compact spaces. 
Proposition 3.2 describes the abelian case while Proposition 3.1 gives a somewhat stronger result in the nonabelian case.

Let $V=\mathbb{F}_{2}[\mathfrak{m}]$ be an $\mathbb{F}_{2}$ vector space whose basis is $\mathfrak{m}$. Let $G(V)$ be the subgroup of the automorphism group of $V$ generated by automorphisms induced by those permutations of $\mathfrak{m}$ which fix all but finitely many elements of $\mathfrak{m}$. Let $G$ be a semidirect product $V \rtimes G(V)$. Note that the cardinality of $G$ is $\mathfrak{m}$.

Proposition 3.1. If $K$ is a compact space then $G$ is not isomorphic to a subgroup of $\pi_{1} K$.

Proof. Suppose to the contrary that we have $G \subseteq \pi_{1} K$, up to isomorphism. We fix two constant $\mathfrak{m}$-sequences $(c)$ and $(d)$ in $\mathfrak{m}$ such that $c \neq d$. By Proposition 2.2 we have two $\mathfrak{m}$-sequences $\left(a_{\tau}\right)$ and $\left(b_{\tau}\right)$ in $\mathfrak{m}$ and an $s \in v \mathfrak{m} \backslash \mathfrak{m}$ such that for each $\tau<\mathfrak{m}$ the elements $a_{\tau}, b_{\tau}, c$ and $d$ are pairwise distinct but $\lim _{s} a_{\tau}=\lim _{s} b_{\tau}$. Let $g_{\tau} \in G(V) \subseteq G$ be those elements which induce by conjugation a cyclic permutation of $\left(a_{\tau}, b_{\tau}, c, d\right)$. If $g=\lim _{s} g_{\tau}$ then properties (i) and (ii) of $\lim _{s}$ imply that $g$ induces a cyclic permutation of $\left(\lim _{s} a_{\tau}, \lim _{s} b_{\tau}, c, d\right)$. Since $\lim _{s} a_{\tau}=\lim _{s} b_{\tau}$ this is possible only when all the elements of this quadruple are equal. Since $c \neq d$ we have a contradiction.

The group $G$ in Proposition 3.1 cannot be abelian since any abelian group can be embedded in a product of copies of $\mathbb{Q}$ and $\mathbb{Q} / \mathbb{Z}$. Since the cardinalities of $\mathbb{Q}$ and $\mathbb{Q} / \mathbb{Z}$ are nonmeasurable Corollary 4 in $[6]$ implies that they are the fundamental groups of some compact spaces, hence so are their products. Still, we have the following.

Proposition 3.2. There exists an abelian group $A$ which is not isomorphic to $\pi_{1} K$ for any compact $K$.

Proof. Proposition 3.1 in [8] says that there exists an abelian group containing a subgroup $A$ which is $\mathfrak{m}$-pure but not $\mathfrak{m}^{+}$-pure $\left(\mathfrak{m}^{+}\right.$denotes the successor cardinal of $\mathfrak{m}$ ).

Suppose that $A=\pi_{1} K$ for some compact space $K$. By Proposition 1.2 we have an m-limit defined on $A$. Proposition 5.4 in [8] says that if $A$ admits $\mathfrak{m}$-limits then $A$ is $\mathfrak{m}^{+}$-pure in any group containing it as an $\mathfrak{m}$-pure subgroup. We have obtained a contradiction.

As an immediate corollary of Proposition 3.1 or Proposition 3.2 we obtain:

THEOREM 3.3. The following statements are equivalent:

(i) There exists a measurable cardinal.

(ii) There exists a group which cannot be realized as the fundamental group of a compact space. 
The following class of groups has been communicated to the author by Eda [2]. Let $G=\langle I \mid \mathcal{R}\rangle$ be a group with generators $I$ and relations $\mathcal{R}$. Each element of $\mathcal{R}$ is a finite word on letters $i$ and $i^{-1}$ where $i \in I$. Suppose that

(*) For each $i \in I$ the cardinality of the subset

$$
\mathcal{R}_{i}=\left\{R \in \mathcal{R} \mid i \text { or } i^{-1} \text { appears in } R\right\}
$$

is nonmeasurable.

Proposition 3.4. Each group $G$ as above is the fundamental group of a compact space.

Proof. Let $\sim$ be the least equivalence relation on $I$ such that $i \sim k$ if there is a word $R \in \mathcal{R}$ which contains $i$ or $i^{-1}$ and $k$ or $k^{-1}$. Condition $(*)$ implies that the equivalence classes of $\sim$ are nonmeasurable hence $G$ is a free product of nonmeasurable groups $G_{j}$ where $j$ runs through the set $J$ of equivalence classes of relation $\sim$.

By Corollary 4 in [6] there are compact spaces $K_{j}$ such that $G_{j}=\pi_{1} K_{j}$. Fix a cardinal $\kappa$ and embeddings $f_{j}: K_{j} \rightarrow I^{\kappa}$ such that each $f_{j}$ takes the base point to the constant sequence $(1 / 2)$ and its range is contained in $[1 / 2,1]^{\kappa}$. Let $J^{\bullet}=J \cup\{\infty\}$ be the one-point compactification of $J$ considered as a discrete space. The subspace of $I^{\kappa} \times J^{\bullet} /\{(0)\} \times J^{\bullet}$ which is the union of $I^{\kappa} \times\{\infty\}$ and the images of the maps $f_{j}$ and the intervals $[(0),(1 / 2)]$ is compact and its fundamental group is a free product of the groups $G_{j}$, hence is isomorphic to $G$.

\section{Path connected compact spaces}

LEMmA 4.1. If $X$ is a path connected paracompact space of nonmeasurable cardinality then the path components of $\beta X$ are of the form $X$ and $\{x\}$ for $x \in \beta X \backslash X$.

Proof. Theorem 3 in [6] states that $X$ is a path component of $\beta X$, hence if a path $\alpha: I \rightarrow \beta X$ is such that $\alpha(0) \in \beta X \backslash X$ then the whole image of $\alpha$ is contained in $\beta X \backslash X$. By Theorem 2 in [6] this path has to be constant.

THEOREM 4.2. Any group $G$ of nonmeasurable cardinality is the fundamental group of a path connected compact space $Z$.

Proof. Let $K$ be a CW-complex of nonmeasurable cardinality whose fundamental group is $G$. Let $K_{0}=\beta K$ and $f_{0}: D \rightarrow K_{0}$ be a map from a discrete space $D$ to $K_{0}$ such that for each path component $P$ of $K_{0}$ there is exactly one $d \in D$ with $f_{0}(d) \in P$. Let $C\left(f_{0}\right)$ be the mapping cone of $f_{0}$. We define $K_{1}=\beta C\left(f_{0}\right)$. We repeat this process inductively and obtain a sequence $K_{n}, n=0,1,2, \ldots$, of compact spaces and inclusions $i_{n}: K_{n} \hookrightarrow K_{n+1}$.

By Lemma 4.1 we see that $G=\pi_{1} K_{0}$ and $G=\pi_{1} C\left(f_{0}\right)$. Since $K_{0}$ is a compact $C^{*}$-embedded subspace of $C\left(f_{0}\right)$ we see that $\beta C\left(f_{0}\right) / K_{0}$ is homeo- 
morphic to $\beta\left(C\left(f_{0}\right) / K_{0}\right)$. Since $C\left(f_{0}\right) / K_{0}$ is a path connected paracompact space Lemma 4.1 implies that for each $x \in K_{1} \backslash C\left(f_{0}\right)$ the path component of $x$ in $K_{1}$ is $\{x\}$, hence $\pi_{1} K_{1}=\pi_{1} C\left(f_{0}\right)$. By repeating the above argument for each $n=0,1,2, \ldots$ we see that the inclusions $i_{n}: K_{n} \rightarrow K_{n+1}$ induce isomorphisms of the fundamental groups and $i_{n}\left(K_{n}\right)$ is contained in a path component of $K_{n+1}$.

Let $T$ be the telescope of the chain of maps $i_{n}$, that is, the space

$$
\left(\coprod_{n=0}^{\infty} K_{n} \times I\right) /\left(x_{n}, 1\right) \sim\left(i_{n}\left(x_{n}\right), 0\right)
$$

where $x_{n}$ runs over points in $K_{n}$. The telescope $T$ is path connected and its fundamental group is the colimit of $\pi_{1} K_{n}$, which is $G$. Since $T$ is locally compact we can take its one-point compactification $T^{\bullet}=T \cup\{\infty\}$. Let $i: T \hookrightarrow T^{\bullet}$ be the inclusion. Let $p: T \rightarrow[0, \infty)$ be a map which sends $\left(x_{n}, t\right) \in K_{n} \times I$ to $n+t$. Let $g: T \rightarrow I$ be defined as $g(x)=\sin ^{2} p(x)$. Let $\bar{T}$ be the closure of the image of the map $i \times g: T \rightarrow T^{\bullet} \times I$. Since for any positive integer $n$ the space $p^{-1}([0, n])$ is compact we see that $\bar{T}$ has exactly two path connected components: $p^{-1}([0, \infty))$ which is homeomorphic to $T$ and the interval at infinity $\{\infty\} \times I$. The mapping cone of $h:\{0,1\} \rightarrow \bar{T}$ which sends 0 to $T$ and 1 to the interval at infinity is compact and path connected, and has the fundamental group isomorphic to $G$.

\section{References}

[1] W. W. Comfort, On the Hewitt realcompactification of a product space, Trans. Amer. Math. Soc. 131 (1968), 107-118.

[2] K. Eda, private communication, 2003 and 2006.

[3] L. Fuchs, Infinite Abelian Groups, Pure and Appl. Math. 36-I/II, Academic Press, New York-London, 1970/73.

[4] L. Gillman and M. Jerison, Rings of Continuous Functions, Springer, 1960.

[5] A. Kanamori, The Higher Infinite, Springer, 1997.

[6] J. E. Keesling and Y. B. Rudyak, On fundamental groups of compact Hausdorff spaces, Proc. Amer. Math. Soc., to appear.

[7] J. Łoś, Generalized limits in algebraically compact groups, Bull. Acad. Polon. Sci. 7 (1959), 19-21.

[8] C. Megibben, Generalized pure injectivity, in: Symposia Math. 13, Academic Press, London, 1974, 257-271.

Institute of Mathematics

Warsaw University

Banacha 2

02-097 Warszawa, Poland

E-mail: adamp@mimuw.edu.pl

Received 13 February 2006; in revised form 20 March 2006 